\title{
Privatisation et évolution du paysage éducatif indien
}

Privatization and the changing educational landscape in India

Privatización y evolución del paisaje educativo indio

\section{Meera Samson}

Traducteur : Sylvaine Herold

\section{(2) OpenEdition Journals}

Édition électronique

URL : https://journals.openedition.org/ries/9191

DOI : 10.4000/ries.9191

ISSN : 2261-4265

Éditeur

France Education international

\section{Édition imprimée}

Date de publication : 1 décembre 2019

Pagination : $93-101$

ISBN : 9782854206258

ISSN : $1254-4590$

Référence électronique

Meera Samson, "Privatisation et évolution du paysage éducatif indien », Revue internationale d'éducation de Sèvres [En ligne], 82 | décembre 2019, mis en ligne le 01 décembre 2021, consulté le 13 janvier 2022. URL : http://journals.openedition.org/ries/9191 ; DOI : https://doi.org/10.4000/ries.9191 


\title{
Privatisation et évolution du paysage éducatif indien*
}

\author{
Meera Samson ${ }^{1}$ \\ Collaborative Research and Dissemination \\ (CORD), New Delhi
}

En Inde, un grand nombre d'acteurs privés est impliqué dans le secteur éducatif, ce qui n'est guère surprenant dans la mesure où il s'agit d'un marché extrêmement vaste et rentable. Ce sont, pour la plupart, des groupes puissants aux plans social et politique - l'accès au marché ainsi que le fort engagement nécessaire exigent que les acteurs aient de bons contacts et soient capables de négocier le rôle qu'ils entendent jouer. Ces groupes n'ont pas forcément d'expérience directe dans le secteur éducatif, mais peuvent faire appel à des individus ou des groupes qui en ont. Dans cet article, nous traitons des acteurs privés gérant des établissement privés, puis de ceux qui apportent leur contribution aux établissements publics. Enfin, nous abordons brièvement les nouvelles formes de partenariat public-privé en éducation.

\section{L'ENSEIGNEMENT PRIVÉ EN INDE}

Il est courant de parler des établissements d'enseignement privés comme s'ils constituaient un groupe homogène. Cependant, ils diffèrent grandement par leurs caractéristiques fondamentales, s'agissant notamment des capacités des élèves, des infrastructures et des installations. En outre, comme le souligne Fennell (2010), les acteurs privés ont indubitablement des approches très différentes de l'éducation, tirées de leur propre évolution historique et de leur motivation économique. Ce que les établissements d'enseignement privés en Inde ont en commun, c'est qu'ils sont tenus légalement de fonctionner sur une base non lucrative.

\section{Croissance des effectifs des établissements d'enseignement privés}

Le nombre d'inscriptions dans les établissements privés a considérablement augmenté en Inde : le nombre d'élèves de la première à la huitième année d'enseignement est passé de 32 millions en 2007-2008 à 47 millions en 2015-2016 (U-DISE, plusieurs années). Ces chiffres comprennent les inscriptions dans l'ensemble des établissements privés reconnus par le gouvernement - qu’ils soient aidés ou non ${ }^{2}$.

\footnotetext{
* Article traduit par Sylvaine Herold.

1. L'auteure remercie Anuradha De et Claire Noronha pour leurs contributions et commentaires.

2. Certains États ont une forte proportion d'établissements privés aidés par le gouvernement. Cette aide couvre essentiellement les salaires des enseignants. Ces établissements appliquent peu ou pas de frais de scolarité. Les établissements aidés ne peuvent recruter d'enseignants supplémentaires que sur autorisation du gouvernement (De et al., 2002).
} 
Pour la même cohorte, le nombre d'inscriptions dans les établissements publics en Inde, on parle de government schools ou écoles du gouvernement - est passé de 101 millions à 78 millions.

Les établissements privés non aidés par le gouvernement couvrent leurs coûts par le biais du paiement de frais de scolarité par les parents et sont en grande partie libres de définir leurs propres politiques d'admission et de recrutement des enseignants, ainsi que le curriculum et le niveau de frais de scolarité qu'ils appliquent. L'un des aspects qui contribue à les rendre extrêmement populaires en Inde est qu'ils sont associés à l'enseignement de la langue anglaise, considérée comme essentielle pour obtenir un emploi bien rémunéré.

Auparavant, les établissements privés non aidés pourvoyaient essentiellement aux besoins des élites des zones urbaines. Il s'agissait d'établissements dotés d'infrastructures et d'installations bien développées et d'enseignants hautement qualifiés, proposant un enseignement de la maternelle à la $12^{\mathrm{e}}$ année - un service inabordable pour la majorité de la population. Cependant, depuis les années 1990, une vaste gamme d'établissements a été créée afin de répondre à la demande du public, s'adressant à toutes les strates de la population. Cela comprend les écoles bon marché, qui fonctionnent essentiellement en tant qu'établissements non reconnus ${ }^{3}$, dispensant des cours dans des salles de classe exiguës et bondées, et employant des enseignants mal payés et peu qualifiés.

La croissance rapide des inscriptions dans le secteur privé est à relier au sous-financement du système éducatif public. Si des investissements ont été réalisés dans le secteur des établissements publics, notamment par le biais de programmes financés par le gouvernement central, l'essentiel des dépenses en éducation provient des budgets des État, et la plupart ${ }^{4}$ de ces derniers opèrent dans un contexte de grave pénurie de ressources. Cette pénurie de ressources apparaît clairement si l'on considère le déficit de recrutement d'enseignants dans les établissements publics.

Dans le même temps, la croissance des établissements privés a été alimentée par l'attrait d'opportunités d'affaires pour les acteurs nationaux et transnationaux. Cela s'est observé très clairement au cours des deux décennies passées, en particulier dans le secteur des écoles privées bon marché.

\section{Les écoles privées bon marché}

Le discours autour des écoles privées bon marché (low-fee private schools) a évolué au fil du temps. Ces établissements ont d'abord été encouragés car promouvant l'équité, en offrant aux pauvres une éducation de qualité. James Tooley (2009) en est l'un des principaux promoteurs. Le plaidoyer en faveur de ces écoles, également appelées budget schools [écoles économiques], a conduit au financement de recherches visant à comparer les niveaux d'apprentissage dans les établissements publics et bon marché, et à montrer à quel point ces derniers étaient efficaces et peu coûteux. Ces établissements ont été promus par des groupes à l'idéologie pro-marché

3. Les établissements privés non aidés doivent répondre à certains standards afin d'être reconnus par le gouvernement, bien que ces standards aient considérablement varié d'un État à l'autre par le passé.

4. La dépense par élève consacrée aux dépenses élémentaires par les gouvernements des États varie considérablement. En 2014-2015, elle était de 39343 roupies [environ 500 euros] dans l'Himachal Pradesh et de 5298 roupies [environ 67 euros] dans le Bihar (Kingdon, 2017). 
- prônant le choix scolaire et les chèques éducation. La philosophie sous-jacente était que la concurrence conduirait à une éducation de meilleure qualité. Les arguments de Tooley en faveur de l'efficacité des écoles bon marché ont suscité un vif intérêt, bien que plusieurs spécialistes aient contesté la validité de ces affirmations ${ }^{5}$. L'un des facteurs ayant contribué à rendre ces établissements attractifs pour les parents à revenu modeste était leur prétention à enseigner l'anglais. Mais cela également a été remis en cause, les enseignants étant eux-mêmes incapables de parler anglais couramment.

Au fil du temps, ces écoles bon marché se sont transformées en de véritables entreprises éducatives ( $e d u$-business) avec l'entrée du capital de sociétés. Les projections pour le secteur prévoyaient une forte rentabilité, en particulier pour des chaînes d'établissements proposant un enseignement standardisé. Les écoles Rumi et les Indus Academies sont deux exemples de chaînes de ce type créées en Inde (Garg, 2011 ; Nambissan, 2013). Srivastava (2016) les considère comme une nouvelle vague, dans laquelle les écoles bon marché sont associées à des fournisseurs de services connexes pour le secteur éducatif, comprenant des "systèmes de notation » et des " systèmes de fourniture de curriculum pré-écrits ». Nambissan (2013), quant à lui, se réfère à la standardisation comme à une mesure visant à mettre les établissements "à l'abri des enseignants ", avec l'idée que même des enseignants aux qualifications et à la formation limitées puissent dispenser le curriculum et soient facilement remplaçables en cas de départ. Cette auteure souligne également qu'il existe une interprétation délibérément erronée de ce que doit être une éducation de qualité pour les pauvres. Ce qui est proposé n'est en fait qu'un curriculum minimaliste et dilué, dispensé par des enseignants mal payés et sous-qualifiés.

La loi sur le droit à l'éducation (Right To Education Act) a été adoptée en 2009 et définit des standards pour les infrastructures et les enseignants pour l'ensemble des établissements. Cette loi a joué un rôle important dans la mise en place d'un cadre réglementaire pour les établissements publics et privés en Inde. Les écoles bon marché ne respectant pas ces standards ont dû fermer. Cela a également eu un impact sur la rentabilité des chaînes scolaires (Garg, 2011). Les écoles Rumi et les Indus Academies ont ainsi mis la clé sous la porte. Les acteurs privés ciblent désormais un segment de population ayant une capacité à payer légèrement supérieure, via des « écoles privées abordables » (affordable private schools) (Nambissan, 2013).

\section{Impact de la croissance de l'enseignement privé}

Les arguments généralement avancés en faveur des établissements privés sont connus. Par rapport aux établissements publics, ils sont associés à un enseignement de meilleure qualité et moins coûteux. Les enseignants sont par ailleurs censés évoluer dans un système dans lequel le niveau de responsabilité vis-à-vis de la direction est élevé, direction qui à son tour rend des comptes aux parents payant des

5. L'étude d'Harma (2011) révèle que les familles dont les enfants fréquentent les écoles bon marché ne sont pas pauvres de façon chronique et qu'elles y recourent faute d'avoir pu les inscrire ailleurs pour des raisons financières. Voir également Nambissan (2013) et Srivastava (2016) pour d'autres études contestant les arguments de Tooley et de ses collègues. 
frais de scolarité. Il est important de distinguer les différents types d'établissements privés et nos propos précédents doivent nous faire prendre conscience qu'il existe une grande variété d'établissements privés, que ce soit en termes de qualité, de coût ou de reddition de comptes.

Les inconvénients associés à la promotion des établissements privés sont également connus ; ils ont souvent à voir avec la manière dont ces établissements contribuent à réduire l'équité au sein de la société dans son ensemble, une discrimination des enfants s'opérant entre établissements publics et privés mais également au sein même des établissements privés, en fonction de la capacité financière des parents. L'écart entre filles et garçons observable en ce qui concerne les inscriptions des établissements privés est également bien documenté, avec une proportion plus élevée de garçons inscrits. Il convient également de se montrer attentif aux formes de ségrégation fondées sur d'autres critères. Par exemple, les établissements privés peuvent restreindre les admissions aux enfants d'un groupe social en particulier, avec des conséquences importantes sur l'intégration sociale.

Répétons ici que si l'ensemble des établissements est tenu de fonctionner dans le cadre posé par la Constitution, qui confère un statut laïque à la nation indienne, les établissements publics sont plus susceptibles de le faire que les établissements privés. Ils offrent la possibilité d'une socialisation commune d'enfants d'origine socio-économique différente. Ils sont également tenus de ne pas discriminer les enfants des castes défavorisées. Les établissements privés sont libres d'avoir leur propre point de vue idéologique sur le rôle de l'éducation dans la construction des citoyens. Les établissements Ekal Vidyalaya - des écoles à enseignant unique installées dans des zones tribales et rurales isolées - disent par exemple s'efforcer d'inculquer aux élèves un esprit de " nationalisme » et de " patriotisme ${ }^{6}$, sans jamais se référer à l'éducation comme impliquant un respect de la diversité ou un esprit d'inclusion.

Les problèmes d'inégalités et de ségrégation sont pourtant cruciaux dans une société marquée par un haut niveau d'inégalité. L'impact est particulièrement grave pour les enfants situés au bas de l'échelle sociale et économique, qui risquent le plus d'être exclus des établissements privés les plus recherchés. Les établissements non payants sont moins sous pression car ils accueillent de manière croissante des enfants dont les parents ne sont pas en mesure de s'exprimer pour améliorer le système. Il existe en outre un problème d'instabilité et de discontinuité : les familles peuvent ne plus être en mesure de payer les établissements privés en cas de difficultés financières ; les enfants peuvent alors être transférés vers des établissements publics ou abandonner complètement leurs études.

Le message explicite est que le gouvernement ne peut pas renoncer à sa responsabilité de fournir une éducation de qualité à tous ses citoyens. Si les acteurs privés peuvent jouer un rôle, on ne peut pas attendre d'eux qu'ils assument le rôle du gouvernement. Le système scolaire public joue également un rôle crucial pour améliorer la qualité de l’enseignement dans les établissements privés ${ }^{7}$.

6. https://www.ekal.org/

7. Les établissements privés se doivent de surpasser les résultats des établissements publics pour attirer des clients (De et al., 2002). 


\section{LES ACTEURS PRIVÉS APPORTANT LEUR CONTRIBUTION AUX ÉTABLISSEMENTS PUBLICS}

Un autre type de participation des acteurs privés est lié à leur contribution au système éducatif public. Une pression croissante s'exerce sur le gouvernement central et les gouvernements des États pour améliorer les résultats d'apprentissage des élèves, en partie du fait des tests internationaux (par exemple PISA ${ }^{8}$ ) mais aussi nationaux (par exemple $\mathrm{ASER}^{9}$ ), qui ont donné une piètre image du système scolaire indien. Cela a suscité un intérêt à collaborer avec des acteurs du secteur privé disposant d'une expertise en matière d'amélioration des résultats d'apprentissage. Les acteurs privés contribuent également au système éducatif public via d'autres apports, qui ne sont pas directement liés à l'amélioration des résultats d'apprentissage.

Plusieurs questions essentielles se posent, s'agissant de ces contributions : de quelle nature sont-elles et comment cela profite-t-il à l'accès et à la qualité de l'éducation? Qui finance ces initiatives $?^{10}$ Quelles sont les motivations des acteurs privés impliqués? Comment envisagent-ils le rôle de l'éducation?

Notons que le choix des acteurs privés autorisés à apporter leurs contributions au système public est fondamentalement politique, bien que soient le plus souvent évoquées leur expertise et/ou les ressources qu'ils mobilisent. La durée pendant laquelle les initiatives sont autorisées est également une décision politique, qui n'est pas sans conséquence sur leur impact final sur le système. Les acteurs privés rapportent ${ }^{11}$ devoir investir énormément de temps et d'énergie afin de conclure des accords avec le gouvernement, ainsi qu'en cours de projet. Il existe différents types d'initiatives.

\section{Initiatives financées essentiellement par le gouvernement}

Parmi les premières contributions des acteurs privés figure la formation à des compétences spécifiques, comme l'utilisation des ordinateurs et l'enseignement de l'anglais. Si les compétences informatiques ont été apportées par des acteurs nationaux et internationaux, tels que la Fondation Azim Premji ou Microsoft, l'enseignement de l'anglais est principalement assuré par des groupes britanniques ${ }^{12}$. Ces services étaient principalement achetés par le gouvernement.

8. PISA : programme international pour le suivi des acquis des élèves. L'enquête PISA a été menée dans les États de l'Himachal Pradesh et du Tamil Nadu en Inde en 2009, ces deux États étant réputés disposer d'un système éducatif public opérationnel.

9. ASER est l'acronyme pour Annual Survey of Education Report [enquête annuelle sur l'état de l'éducation], une enquête qui teste chaque année les apprentissages des enfants dans des ménages à travers toute l'Inde.

10. Les sources de financement comprennent les fonds RSE des entreprises (qui découlent d'une politique gouvernementale imposant aux grandes entreprises de verser $2 \%$ de leurs bénéfices à la responsabilité sociale des entreprises), ainsi que des fonds provenant d'autres personnes et d'autres groupes.

11. Sur la base d'interactions avec plusieurs groupes privés ayant apporté des contributions au gouvernement.

12. Le British Council travaille en partenariat avec les gouvernements des États dans toute l'Inde pour permettre aux enseignants et aux formateurs de formateurs de gagner en confiance et de maîtriser l'anglais, aux niveaux primaire et secondaire. 


\section{Initiatives financées principalement par les acteurs privés}

Ces initiatives sont de différents types. Elles portent sur l'amélioration des infrastructures et des installations (y compris la rénovation des salles de classe et des toilettes, ainsi que la mise à disposition de bibliothèques, d'ordinateurs et de laboratoires scientifiques) ; de tels apports sont très appréciés dans les nombreux établissements scolaires sous-financés;

Elles concernent également la formation des enseignants, notamment aux nouvelles pédagogies et à la création d'environnements propices à l'apprentissage. Ces interventions peuvent avoir un impact positif sur le système, en particulier si la formation est dispensée par des formateurs de formateurs ayant une bonne compréhension du contexte dans lequel les enseignants évoluent. Les enseignants ont néanmoins souvent le sentiment que leur situation est mal comprise ${ }^{13}$.

Certains groupes mettent également à disposition des enseignants volontaires pour des durées variables. Teach For India, inspiré de Teach For America, a par exemple suscité un intérêt considérable. Ce groupe propose à de jeunes enseignants volontaires, instruits et généralement issus de milieux privilégiés, de travailler pendant deux ans dans des établissements aux ressources limitées, dans le but affiché d'éliminer les inégalités en matière éducative ${ }^{14}$. De tels programmes ont un impact sur le système, dans la mesure où ils bénéficient d'une forte visibilité et de la promotion de l'impact de ces initiatives.

Tous ces programmes sont susceptibles d'impacter de manière très significative le secteur éducatif à long terme, dans la mesure où ils contribuent à la manière dont le curriculum est négocié, c'est-à-dire les méthodes d'enseignement utilisées, ainsi que la manière et la fréquence à laquelle les enfants sont évalués.

En outre, plusieurs groupes ont investi dans l'amélioration du leadership, dispensant des formations aux directeurs d'établissement ainsi qu'aux autorités des circonscriptions scolaires ${ }^{15}$. Ces apports en termes de gestion ont un impact très significatif sur le secteur éducatif, dans la mesure où ils impliquent la définition d'objectifs et de priorités à l'échelle des établissements et du système scolaire dans son ensemble.

Un certain nombre d'acteurs privés ont contribué au développement du curriculum en collaborant étroitement avec les Conseils d'État pour la recherche et la formation en éducation (State Councils of Educational Research and Training). Cela s'inscrit généralement dans le cadre de leurs autres apports au secteur éducatif de ces États, apports qui ont un impact très significatif sur l'éducation.

La plupart de ces initiatives sont pressées de démontrer leur impact sur les résultats d'apprentissage. Du côté des acteurs privés, il s'agit de montrer des résultats au gouvernement et à leurs donateurs ; du côté du gouvernement, il s'agit de montrer

13. Propos rapporté par des enseignants lors d'études menées par le groupe de recherche indépendant CORD (Collaborative Research and Dissemination).

14. Teach For India a été critiqué pour ne dispenser qu'une formation de courte durée aux enseignants volontaires : les jeunes enseignants suivent d'abord une formation intensive de cinq semaines, puis reçoivent le soutien continu d'un superviseur.

15. L'Inde est une république fédérale composée de 29 États et 7 Territoires de l'Union, eux-mêmes divisés en districts, puis en tehsils portant différents noms selon les États (dont les « blocs » en zones rurales, pour «bloc de développement communautaire », regroupant plusieurs villages). (NdT) 
des signes d'amélioration aux électeurs et au monde entier. Conséquence négative, les acteurs privés sont poussés à jouer le jeu du « avant »/ « après », par lequel les faiblesses du système public avant l'initiative et les améliorations en résultant sont accentuées. Cette inefficacité projetée nuit à l'image de l'administration et des enseignants du secteur public.

Il existe enfin des initiatives financées par le gouvernement, avec un financement complémentaire de donateurs (fourniture de repas scolaires) : tous les établissements publics indiens sont tenus d'offrir un repas de midi aux élèves de la première à la huitième année d'enseignement. Pour cela, le gouvernement a noué des liens avec de nombreux acteurs privés - certains étant entièrement financés par le gouvernement, d'autres apportant un financement complémentaire.

Un acteur privé est particulièrement visible, qui apporte des financements complémentaires provenant d'un grand nombre d'entreprises donatrices, nationales et internationales : il s'agit d'Akshay Patra, une vaste entité unique qui a mis en place des cuisines mécanisées ${ }^{16}$ et fournit des repas à très grande échelle (servant plus de 1,7 million d'écoliers chaque jour). Dirigée par le groupe Iskcon ${ }^{17}$, la société est actuellement présente dans dix États. Si ce groupe a reçu de nombreuses récompenses pour son travail, il a également fait la une des médias en 2018 pour son refus d'utiliser de l'ail et de l'oignon dans ses plats à Bangalore, bien que l'oignon figure sur la liste des ingrédients spécifiés par le gouvernement. Selon ses détracteurs, en imposant ainsi un code alimentaire brahmanique à des écoliers qui ne sont pour la plupart pas issus des castes supérieures, l'action du groupe a un impact négatif sur le nombre d'enfants qui mangent à l'école.

Le passage en revue des différentes contributions des acteurs privés aux établissements publics indiens nous a permis de constater à quel point celles-ci sont variées. Il est absolument nécessaire que ces contributions soient examinées de manière critique par le gouvernement et la société civile dans son ensemble, s'agissant à la fois de leurs aspects positifs et négatifs.

\section{Partenariats PUblic-PrivÉ}

Ces quinze dernières années, la politique du gouvernement a également évolué pour permettre d'autres formes de privatisation, connues sous le terme de partenariats public-privé (PPP). L'idée de ces partenariats est d'introduire de meilleures techniques pédagogiques et de gestion, et de permettre un certain degré de partage des risques entre les secteurs public et privé. Les PPP sont censés fonctionner au même niveau de financement que les établissements publics et permettre ainsi de démultiplier l'offre scolaire. Ils sont également censés respecter des standards de performance stricts et accroître ainsi le niveau de responsabilité dans le système (Chaudhry et Uboweja, 2014). Nous abordons dans ce qui suit différents types de PPP existant actuellement en Inde.

16. Bien que son principal atout réside dans sa capacité à gérer de grandes cuisines centralisées dans des zones urbaines et semi-urbaines, la société gère désormais également des cuisines décentralisées dans quelques zones rurales.

17. International Society for Krishna Consciousness [Association internationale pour la conscience de Krishna, appelée communément « Hare Krishna »]. 


\section{Établissements publics gérés par le privé}

Dans cette première modalité, le gouvernement contracte directement avec des prestataires privés pour exploiter des établissements publics. L'élément commun ici est la répartition des rôles entre le gouvernement fournisseur d'infrastructures et le secteur privé gestionnaire. Les coûts de fonctionnement sont partagés selon des modalités différentes en fonction des cas. Il existe de nombreuses variantes de ce modèle. Nous en abordons deux.

\section{Les établissements fonctionnant avec les enseignants en poste}

Dans cette modalité de mise en œuvre des PPP, dite « adoption d'établissement ", les acteurs privés concluent des accords avec le gouvernement afin d'adopter des établissements publics. Ils sont libres d'utiliser le terrain, le bâtiment et les enseignants de l'établissement, mais doivent couvrir les coûts de fonctionnement. Les élèves continuent de ne payer aucun frais. Certains établissements au Rajasthan sont gérés selon ce modèle par la Bharti Foundation. Cet acteur privé reçoit des financements complémentaires provenant de donateurs afin de couvrir les coûts.

\section{Les établissements libres de recruter leurs propres enseignants}

Ici, le gouvernement fournit l'infrastructure scolaire et rembourse certains coûts sur la base d'un montant déterminé par élève. Les acteurs privés sont libres de recruter des enseignants, d'utiliser leur propre curriculum, mais on peut leur demander de démontrer l'amélioration des résultats d'apprentissage des élèves. En Inde, certains établissements de Mumbai, comme les établissements gérés par la Fondation Akanksha et d'autres groupes, utilisent ce modèle de PPP. Ils dépendent des dons privés pour combler leurs besoins de financement.

\section{Établissements construits et gérés par le privé}

Le gouvernement utilise désormais une autre modalité de PPP, dans laquelle le partenaire privé construit, est propriétaire et exploite une infrastructure scolaire, le gouvernement prenant en charge une partie des coûts. Le programme des «écoles modèles " (Model School) du gouvernement central indien entre dans cette catégorie : dans le programme Adarsh Model School au Punjab, géré par la Fondation Bharti, le gouvernement fournit le terrain en bail longue durée, $50 \%$ des dépenses en capital et $70 \%$ des frais de fonctionnement par enfant. Les acteurs privés sont autorisés à couvrir leurs coûts via l'admission d'une certaine proportion d'enfants s'acquittant de frais de scolarité, tout en étant libres de recruter des enseignants et de choisir le curriculum.

À l'heure actuelle, cette modalité de PPP est encore limitée à quelques États, mais soulève des questions similaires à celles posées dans la section précédente : qui sont les acteurs privés autorisés à participer? Quelles sont leurs motivations ? Quels 
sont les avantages pour le système éducatif et la société dans son ensemble ? Quel peut être l'impact à long terme ? Il est important d'avoir une vision nuancée de ces PPP.

En Inde, la croissance substantielle des inscriptions dans les établissements privés a certes un impact positif sur l'élargissement de l'accès à une éducation de qualité. Mais trop peu d'attention est accordée aux impacts négatifs que cela peut avoir sur la qualité de l'éducation, ainsi que sur l'équité et la ségrégation dans une société caractérisée par de fortes inégalités sociales. La participation des acteurs privés au système scolaire public progresse également. De nouveau, le débat porte sur la manière dont ces initiatives affectent la vie des enfants issus des groupes défavorisés. Si certaines de ces contributions peuvent s'avérer très utiles, il convient de se demander quels pourraient être les inconvénients de l'ouverture du système éducatif public à des individus et groupes privés, et si cela devrait faire l'objet d'une réglementation. En fin de compte, il incombe au gouvernement de veiller à ce que tous les enfants aient accès à une éducation de qualité, qui leur permette de devenir de bons citoyens. Cela est particulièrement vrai pour les enfants issus des milieux défavorisés, qui ne sont pas en mesure de défendre leurs propres intérêts.

\section{RÉFÉRENCES BIBLIOGRAPHIQUES}

DE A., NORONHA C et SAMSON M . (2002). «Private Schools for Less Privileged: Some insights from a Case Study ». Economic and Political Weekly, n 37 (52).

CHAUDHRY S. et UBOWEJA A.(2014). « Public-Private Partnerships in School Education: Learning and Insights for India ». Working Paper, Central Square Foundation et FICCI.

FENNELL S. (2010). « Public Private Partnerships and Educational Outcomes for the Poor: New Conceptual and Methodological Approaches ». RECOUP Working Paper, $\mathrm{n}^{\circ}$ 37, Université de Cambridge.

GARG N. (2011). « Low Cost Private Education in India: Challenges and Way Forward ». Mémoire de master, MIT Sloan School of Management.

HARMA J. (2011). « Low Cost Private Schooling in India: Is it Pro-poor and Equitable? ». International Journal of Educational Development, $\mathrm{n}^{\circ}$ 31, p. 350-356.

KINGDON G.G. (2017). « The emptying of public schools and growth of private schools in India ». Report On Budget Private Schools In India.

NAMBISSAN G.B. (2013). "Low-Cost Private Schools for the Poor in India: Some Reflections ». India Infrastructure Report 2012, New Delhi et Londres : Routledge.

SRIVASTAVA P. (2016). «Questioning the Global Scaling-up of Low-fee Private Schooling: the nexus between business, philanthropy, and PPPs ». In A. Verger, C. Lubienski et G. Steiner-Khamsi (dir.), The Global Education Industry. World Yearbook of Education 2016. Routledge, p. 248-263.

TOOLEY J. (2009). The Beautiful Tree: A Personal Journey into how the World's Poorest People are Educating Themselves. New Delhi : Penguin.

U-DISE (plusieurs années). Elementary Education in India: Trends. Government of India and National University of Educational Planning and Administration. 